\title{
Outline- and solid-angle orientation illusions have different determinants
}

\author{
PETER WENDEROTH and TONY O'CONNOR \\ University of Sydney, Sydney, New South Wales, Australia
}

\begin{abstract}
Six experiments together suggest that negative illusions (assimilation effects) induced by either single solid acute-angle or double solid-angle (Bourdon) displays are explained by a failure to discriminate the judged edge of the angle(s) from the other edge and from the average orientation of the display [the bisector(s) of the angle figure]. The evidence for this is that Bourdon effects occur only when the judged edge is straight (Experiments 1 and 2) and only when the edge is not vertical or horizontal (Experiments 1, 3, and 4), and that these effects are enhanced by manipulations that reduce acuity for parallelism (Experiments 5 and 6). None of these variables affect outline-angle tilt illusions in the same fashion, which strongly suggests that solid- and outline-angle illusions have entirely different determinants.
\end{abstract}

If a solid acute angle is mirror-reflected about its vertex so that one angle arm and its reflection form a straight edge (ACB in Figure 1a), the edge appears bent in the same direction as the chevron formed by other angle arms (DCE). This is the Bourdon illusion, recently studied by Rozvany and Day (1980) and Wenderoth, O'Connor, and Johnson (1986b). In one of their experiments, Wenderoth et al. (1986b) required observers to complete the three tasks shown in Figure 1. With all comparison lines pivoting around point 0 , the tasks were, first, to set ${ }^{\circ} C^{\prime}$ parallel to $\mathrm{BC}$ (Figure 1a); second to set $\mathrm{OC}^{\prime \prime}$ parallel to $\mathrm{AC}$ (Figure $1 \mathrm{~b}$ ); and third, to set the chevron, $\mathrm{C}^{\prime} \mathrm{OC}$ " to match the chevron BCA (Figure 1c).

These same judgments also were obtained using an outline-angle display (Figure 1d). Parallel matches to outline figures exhibited illusory errors consistent with angle expansion (positive effects), so that $0 C^{\prime}$ was set too far counterclockwise relative to a pretest match to a single line at $\mathrm{BC}$; $\mathrm{OC}$ " was set too far clockwise. On the other hand, matches to solid angles showed errors consistent with angle contraction (negative effects): $0 C^{\prime}$ and $0 C^{\prime \prime}$ were set too far clockwise and counterclockwise, respectively. Chevron matches were not predictable from the parallel matches. For the outline display, chevron matches were close to zero, whereas chevron matches to the solid display were even more negative than the sum of the individual parallel matches.

On the basis of other evidence from studies of the tilt illusion and aftereffect (Wenderoth \& Johnson, 1984; Harris \& Calvert, 1985), Wenderoth, O'Connor, and Johnson (1986c) interpreted their data as consistent with

This research was supported by the Australian Research Grants Scheme, Grant A28515620 I, to the first author. This grant also provided for the second author's research assistance. We thank John Holden for assistance with computer displays and software and Mike Johnson for helpful discussion.

The authors' mailing address is: Department of Psychology, University of Sydney, Sydney, N.S.W., Australia 2006. the hypothesis that perceptual judgments represent a compromise between separate neural channels that code orientation, position, and collinearity. Thus, in chevron matching to outline displays, the individual angle expansion effects are counteracted by information about the straightness of the whole edge, ACB, and by the positional attraction of $\mathrm{BC}$ and $\mathrm{AC}$ toward the nearby contours $\mathrm{EC}$ and DC. In solid displays, both the orientation and positional effects are negative, and perceptually it is difficult to isolate the edge, ACB, from the rest of the figure. Hence, all of these effects reinforce each other in the chevron task, resulting in a large negative illusion.

The experiments reported here arose from a series of informal observations. First, when we ourselves matched chevrons to solid displays, the proposed compromise nature of the judgment was evident: after setting the chevron so that its upper and lower arms appeared parallel to the edges AC and BC (which is how most subjects say they attempt the task), the chevron appeared not quite so bent as the edge formed by the solid angles. Thus, judgments usually involved a simultaneous upper- and lower-parallel match, followed by an additional negative bending so that the match "looked right." Second, most subjects asked, following the experiment, whether all the edges had been straight in fact, even though they had been instructed otherwise.

For these reasons, we decided to repeat the earlier experiments, using outline and solid displays in which the edges, ACB, actually were bent, that is, in which the triangle BCE (Figure 1) not only was reflected but also rotated around $C$. In this way, we hoped to remove cues to collinearity that might otherwise be confounded with illusions of orientation and position.

\section{EXPERIMENT 1}

The aim of this experiment was to obtain parallel and chevron matches, as in Figure 1, to outline and solid dis- 


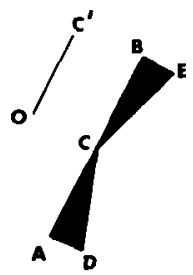

b

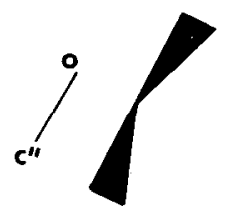

C

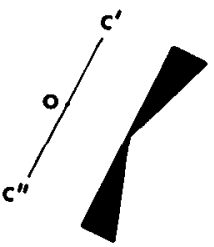

d

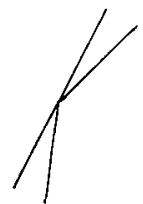

Figure 1. Stimuli and tasks used by Wenderoth et al. (1986a) to study the Bourdon illusion. (a) Solid Bourdon figure; task is to set OC' parallel to BC. (b) Task is to set $\mathrm{OC}^{\prime \prime}$ parallel to $\mathrm{AC}$. (c) Task is to set chevron $\mathrm{C}^{\prime} \mathrm{OC}^{\prime \prime}$ to match edge ACB. (d) Outline version of the Bourdon display.

plays in which the edge, $\mathrm{ACB}$, was not straight so that the angle ACB was not $180^{\circ}$ but $160^{\circ}$.

\section{Method}

Apparatus. Stimuli were displayed on the flat screen of a Tektronix 608 monitor (P4 phosphor), interfaced with a Hewlett-Packard 1350A graphics translator and a PDP-11/20 computer. The subject used the outer pair of three microswitches to rotate lines $0 \mathrm{C}^{\prime}$ or ${ }^{\circ} \mathrm{C}^{\prime \prime}$ (Figure 1) clockwise or counterclockwise or to rotate them simultaneously in opposite directions when a chevron, $C^{\prime} 0 C^{\prime \prime}$, was displayed. A press on the third, central, microswitch indicated completion of adjustment and caused the computer to remove the display, store the result and initiate the next stimulus, either immediately (outline angles) or after about $11 \mathrm{sec}$ (solid figures). The display was viewed in a dark, windowless laboratory, adjacent to the experimenter's room, from a padded forehead- and chinrest $57 \mathrm{~cm}$ from the screen, so that $1 \mathrm{~cm}$ on the screen subtended $1^{\circ}$ of visual angle.

Stimuli. All the displays were derived from acute angles subtending $12.5^{\circ}$, which produced largest effects in the Rozvany and Day (1980) Bourdon illusion studies, and which lay in between the two angle sizes used by Wenderoth et al. (1986b), that is, $10^{\circ}$ and $20^{\circ}$. Using the conventional Cartesian coordinate system (horizontal, $0^{\circ}$; first quadrant at upper right), the orientations of the four angle arms were fixed at $\mathrm{CE}, 4^{\circ} ; \mathrm{CB}, 57.5^{\circ} ; \mathrm{CA}, 257.5^{\circ}$; and $\mathrm{CD}, 270^{\circ}$. (It had been intended to orient CB at $67.5^{\circ}$, where Rozvany and Day had found greatest illusions, but a programming error caused the $10^{\circ}$ shift). All angle arms were $1^{\circ}$ long so that the total edge, $A C B$, was $2^{\circ}$ in length. Each arm of the chevron $\left(C^{\prime} O C^{\prime \prime}\right)$ was $0.8^{\circ}$ long, and the orthogonal distance, $\mathrm{OC}$, was $0.6^{\circ}$. Solid angles were produced by 50 cross-hatch vectors, each $0.1 \mathrm{~mm}$ thick, separated by $0.1 \mathrm{~mm}$.

One buttonpress rotated $0 C^{\prime}, 0 C^{\prime \prime}$, or both, by a step of $0.25^{\circ}$, and continuous pressing produced apparent movement of about $2 \% \mathrm{sec}$. Stimuli were viewed through a black circular outline mask and partially crossed Polaroid filters, so that line luminance was about $1.8 \mathrm{~cd} / \mathrm{m}^{2}$ measured by an S.E.I. photometer. Contrast, defined as $\left(L_{\max }-L_{\min }\right) /\left(L_{\max }+L_{\min }\right)$, was close to 1.0 .

Procedure. The subjects made 36 judgments: each of nine displays was judged four times, from starting positions for $0 C^{\prime}, 0 C^{\prime \prime}$, or both, of $\pm 2.5^{\circ}$ or $\pm 5^{\circ}$ from true parallelism with respect to edges CB, CA, or both (Figure 1). Three of the nine displays were solid, three were outline, and the remaining three were pretest (control) conditions in which only single lines were presented at CB and CA. Bracketing prior to final setting was encouraged. In every case, illusions were measured as test minus pretest settings, so that errors consistent with angle expansion were positive whereas errors consistent with angle contraction were negative.
Subjects. There were 18 subjects, drawn from an introductory psychology course, who received nominal course credit in return for participation. All had emmetropic or corrected vision.

\section{Results}

The results are shown in Table 1 , in which illusions are the difference between test and pretest measures and the mean chevron errors are the deviation of one chevron arm from parallelism.

Three aspects of the results were of particular interest. First, the chevron matching error to the solid figure $\left(-0.07^{\circ}\right)$ was not significantly different from zero $[t(17)$ $=-0.26, p>.05]$. Second, although the upper parallel (UP) match to the solid angle was significant and negative $\left[-1.14^{\circ} ; t(17)=-4.30, p<.001\right]$, the lower parallel (LP) match to the same display was not significant $\left[+0.14^{\circ} ; t(17)=0.53, p>.05\right]$. Third, not only were the UP and LP errors significant and positive for the outline display $\left[+1.08^{\circ}\right.$ and $+1.14^{\circ}$, respectively; $t \mathrm{~s}(17)=4.08$ and $4.30, p<.001$ in both cases], but the chevron (C) match was also significantly positive $\left[+1.63^{\circ} ; t(17)=6.15, p<.0005\right]$.

\section{Discussion}

The nonsignificant $\mathrm{C}$ error for the solid display raises the question as to whether this previously reported negative Bourdon illusion occurs only when the edge to be matched is physically straight (Wenderoth et al., 1986b) or when the task is to adjust it to appear straight (Rozvany \& Day, 1980). The nonsignificant LP error, compared with the significant and negative UP error, raises the further question of whether negative parallel matches to single solid angles occur only when the angle arm to

\section{Table 1}

Means (M) and Standard Errors (SE), in Degrees, of Illusions Obtained in Experiment 1 for Upper Parallel (UP), Lower Parallel (LP), and Chevron (C) Matches to Solid and Outline Displays

\begin{tabular}{lrrrrrrr}
\hline & \multicolumn{3}{c}{ Solid } & & \multicolumn{3}{c}{ Outline } \\
\cline { 2 - 4 } \cline { 6 - 7 } & \multicolumn{1}{c}{ UP } & LP & C & UP & \multicolumn{1}{c}{ LP } & \multicolumn{1}{c}{ C } \\
\hline M & -1.14 & +0.14 & -0.07 & & +1.08 & +1.14 & +1.63 \\
SE & 0.28 & 0.24 & 0.37 & & 0.25 & 0.25 & 0.20 \\
\hline
\end{tabular}


be matched is oriented relatively obliquely (UP arm orientation: $57.5^{\circ}$ ) rather than relatively close to a main axis of space (LP orientation: $257.5^{\circ}$ ). In the former case, the arm was $32.5^{\circ}$ from vertical; in the latter, it was $12.5^{\circ}$.

Taken together, these two observations, or questions, raise the possibility that there is a fundamental difference between solid-angle effects and other well-known visual illusions of direction (including orientation illusions). For example, it is well documented that visual illusions, such as the Zöllner and Poggendorff effects, are larger when the test (judged) component is oriented obliquely rather than vertically or horizontally. Nevertheless, significant illusions still occur when the test segments are vertical or horizontal (Judd \& Courten, 1905; Leibowitz \& Toffey, 1966; Oyama, 1960, 1975; Wallace, 1964; White, 1975). In the case of solid-angle illusions, Rozvany and Day (1980) obtained a near-zero Bourdon illusion when the judged edge was horizontal and a small (about $-0.5^{\circ}$ ) error in setting one edge of the chevron when the edge was vertical. This finding, together with the fact that the LP match was not significant in Experiment 1, may indicate that solid-angle illusions are not merely reduced near the main axes of space, but are abolished.

If this is so, the following hypothesis seems worthy of consideration. The Zöllner illusion and related effects possibly occur due to lateral neural interactions between orientation-selective channels in the visual cortex (see Wenderoth, O'Connor, \& Johnson, 1986c). At least in central vision, there is anisotropy between the number and, perhaps, selectivity of orientation-selective cells tuned to the main axes of space as compared with those that are obliquely tuned, with the latter being fewer in number and, perhaps, more broadly tuned (Bauer, Dow, \& Vautin, 1980; Bouma \& Andriessen, 1968; Campbell \& Kulikowski, 1966; Howard, 1982; Mansfield, 1974; Mansfield \& Ronner, 1978). Hence, the Zöllner illusion and related effects are larger when the test component is oblique because cells responsive to the test segment receive inhibition from a greater range of orientations (they are more broadly tuned) and there also are more cells sending inhibitory signals from orientations nearer the main axes where the inducing components of the illusory figure are located. In brief, orientation illusions such as the Zöllner, and perhaps the Poggendorff to some extent (Wenderoth, O'Connor, \& Johnson, 1986a), involve two components: a basic effect due to neural interactions and a second component due to neural anisotropies that magnify the illusions at or near the obliques.

In these terms, the suggestion that solid-angle illusions do not occur at all near the main spatial axes but are quite robust around the oblique might suggest that these illusions are pure acuity effects: there is a failure to discriminate accurately between the judged edge of the solid angle, the other angle edge, and the angle bisector when the angle is oblique. This does not occur at vertical and horizontal.

Also consistent with the above hypothesis is the failure to obtain any solid-angle effect in Experiment 1 when the chevron was set to match a truly nonstraight chevron. It was noted earlier that informal observation suggested a two-stage matching strategy in which a parallelism setting was made first and then, because the solid edge still "looked more bent," a further adjustment was made. When the solid edge actually is bent, no such second-stage adjustment would occur: just as acuity for nonverticality is high but acuity for departures from true oblique is low (Appelle, 1972; Howard, 1982), so acuity for departures from straightness $\left(180^{\circ}\right)$ is high but acuity for departures from a $160^{\circ}$ angle is low. On this analysis, one would predict no solid-angle chevron illusion in Experiment 1 but that outline-angle effects would occur because they do result from neural inhibitory processes (Wenderoth et al., 1986b), and whether the chevron edge is straight is irrelevant to the occurrence of these processes. Not only did this result occur in Experiment 1, but the outlinechevron effect was much larger than that reported by Wenderoth et al. (1986b) when the test edge was straight. This too is consistent with the hypothesis (Wenderoth et al., 1986b) that the salient collinearity of the outline-angle's edge counteracts the orientation effect: removing this salience by bending the edge thus increases the outlinechevron illusion.

\section{EXPERIMENT 2}

The aim of Experiment 2 was to test directly the hypothesis that solid-figure chevron matches are significantly negative only when the matched edge is physically straight, but that outline-figure positive effects occur both for straight and nonstraight edges. This was done by presenting observers with solid and outline displays in which the matched edge varied from being bent in one direction, through being straight, to being bent in the other direction. This design also allowed us to test whether outline-display illusions would be smaller for straight edges, which provide collinearity cues to counter orientation contrast effects, than for slightly bent edges.

\section{Method}

Apparatus and Procedure. The apparatus and procedure were as for Experiment 1.

Stimuli. There were five basic stimulus figures, shown in Figure 2. We began with a figure resembling a cross (or an hourglass): its axis of symmetry was oriented $45^{\circ}$ and the four angles' arms were $\pm 6.25^{\circ}$ from this axis, at $38.75^{\circ}, 51.25^{\circ}, 219.75^{\circ}$, and $231.25^{\circ}$ (Figure 2C).

The four additional stimuli were generated as follows. Figure 1B differed from Figure $1 \mathrm{C}$ in that the two upper arms were rotated $6.25^{\circ}$ clockwise and the two lower arms rotated $6.25^{\circ}$ counterclockwise. Figure $1 \mathrm{D}$ had equal magnitude but opposite rotations. Finally, Figure 1A differed from Figure 1B by an extra set of $6.25^{\circ}$ rotations, in the same direction; this was also true of Figure 1E as compared with Figure 1D. All acute angles were $12.5^{\circ}$.

Apart from the five outline stimuli shown in Figure 2, there were another five similar, but solid, figures. Adjustable chevrons, identical in size and placement to those in Experiment 1, were located to the left of each stimulus. Hence, Figure 2B (and its solid counterpart) had the truly straight test edge, whereas the straight edge in 


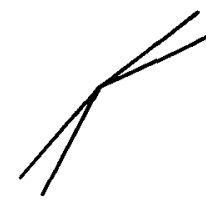

A

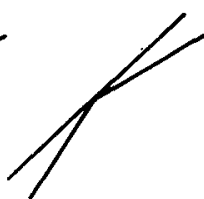

B

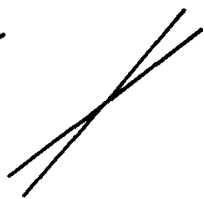

C

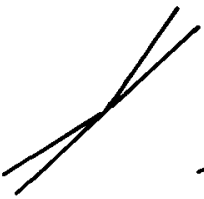

D

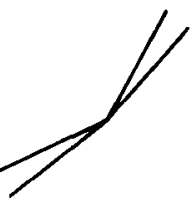

$\mathbf{E}$

Figure 2. Five basic stimulus figures used in Experiment 2. The matching chevron was always to the left of the display so that the only match to a truly straight edge occurred with stimulus B.

Figure 1D was the nonmatched edge. All angle arms subtended $1^{\circ}$ of visual angle.

Subjects. There were 18 subjects, drawn from the same population as those in Experiment 1.

\section{Results}

Mean illusions are shown in Figure 3. The outline illusions induced by the stimuli shown in Figures 2A, 2B, and $2 \mathrm{C}$ were significantly different from zero $[t \mathrm{~s}(17)=$ $7.18,2.82$, and 3.34 , respectively, and $p s<.0005$, $<.02$, and $<.01]$. Illusions induced by the stimuli shown in Figures 2D and 2E were in the expected (positive) direction but were not significant $[\operatorname{ts}(17)=1.74$ and 1.25 , respectively, $p>.05$, although the first of these would be significant on a one-tailed test]. The apparent linear trend across these means was significant $[F(1,238)$ $=17.84, p<.0005$ ], and it was also predicted (see Discussion).

By contrast, the only significant solid-angle illusion was that obtained with the companion stimulus to that shown in Figure $2 \mathrm{~B}$, which had a truly straight edge $[t(17)=$ $-4.36, p<.001]$. Thus, for the solid stimuli, the trend across the means was not linear or quadratic $[F \mathrm{~s}(1,238)$ $=0.88$ and 0.33 , respectively], but both cubic and quartic $[F \mathrm{~s}(1,238)=6.28$ and $12.84, p s<.025$ and $<.0005$, respectively].

The overall mean for outline displays $\left(+1.00^{\circ}\right)$ differed from that for solid displays $\left(-0.28^{\circ}\right)[F(1,238)=46.27$, $p<.0005]$.

\section{Discussion}

The results of this experiment were consistent with the predictions: solid-angle illusions were negative and significant only when the edge to be matched was physically straight. Outline-angle illusions were significant in three of the five conditions (or in four had the tests been onetailed).

The linear trend over the outline-display means was expected to occur as a consequence of the anisotropies of tuning width and density of orientation-selective cells between oblique and near-vertical (or horizontal) axes. Thus, in Figure 2A, the test lines are closer to the oblique than are the inducing lines, so that a large illusion is predicted; in Figure 2E, the reverse is the case, so that a smaller illusion should occur. Similar tendencies were reported by Carpenter and Blakemore (1973, Figure 6). In addi- tion, the data point that fits least well to a linear function in Figure 3 is that for the straight edge match (Figure 2B): this point is too low, again consistent with the view that cues to collinearity when the edge of an outline display is truly straight detract from the orientation contrast effect.

\section{EXPERIMENTS 3 AND 4}

Although these two experiments were conducted separately with different sample sizes, they can usefully be considered together.

It was hypothesized earlier that the magnitude of outlinedisplay effects could be considered as the sum of a basic illusion plus a component reflecting neural anisotropy,

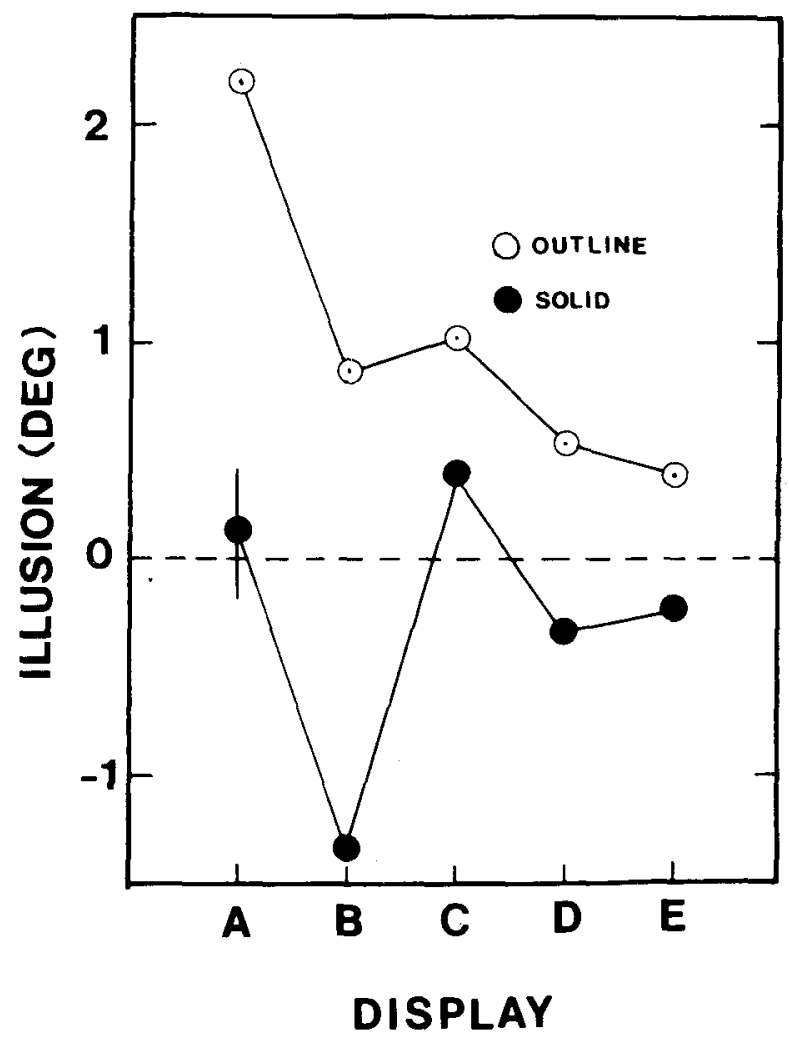

Figure 3. Mean illusions obtained for outline and solid versions of the stimuli shown in Figure 2, Experiment 2. Error bar shows \pm 1 standard error. 
whereas solid-display effects arose solely from a failure to discriminate between various components of the display when it was oriented away from the main spatial axes. On this hypothesis, it can be predicted that outline-display effects will be positive and significant at all orientations of the test edge between horizontal and vertical, but that solid-display effects will not be significant at vertical and horizontal. In Experiments 3 and 4, this prediction was tested using both parallel matching to single solid and outline acute angles (Experiment 3) and using chevron matching to dual-angle outline and solid Bourdon figures (Experiment 4).

\section{Method}

Apparatus and Procedure. The apparatus and procedure were the same as those used in Experiments 1 and 2.

Stimuli. The basic dimensions of all stimuli were as in previous experiments. Single $12.5^{\circ}$-angle stimuli and comparison lines for parallel matching were generated simply by eliminating the lower half of the Bourdon displays (Figure 1). Single angles and Bourdon figures were presented in five different orientations, such that the edge to be matched was oriented $0^{\circ}, 22.5^{\circ}, 45^{\circ}, 67.5^{\circ}$, or $90^{\circ}$. Each subject completed all 60 conditions (5 orientations $\times$ solid, outline, or pretest $\times 4$ starting positions) in a different random order.

Subjects. All subjects were drawn from the population used earlier, with 19 subjects in Experiment 3 (single angles) and 24 subjects in Experiment 4 (Bourdon displays).

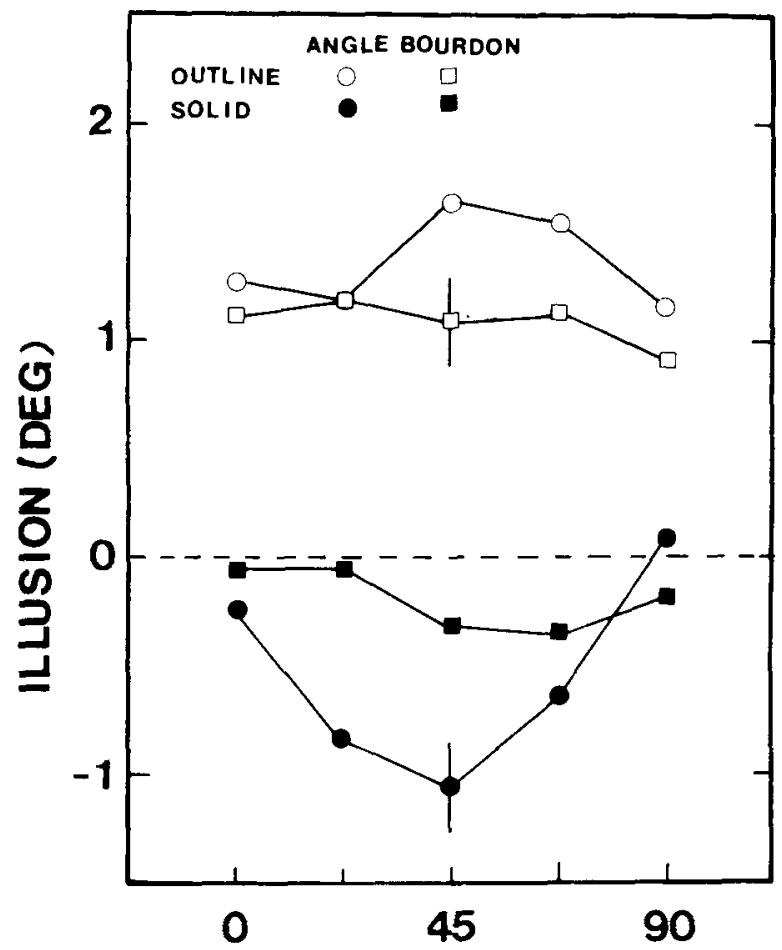

FIGURE TILT (DEG)

Figure 4. Mean illusions obtained for outline and solid single angles (circles, Experiment 3) and outline and solid Bourdon figures (squares, Experiment 4) as a function of test edge orientation $\left(\theta^{\circ}\right.$, horizontal; $90^{\circ}$, vertical). Error bars show \pm 1 standard error for the respective experiments. $N=19$, Experiment $3 ; N=24$, Experiment 4.

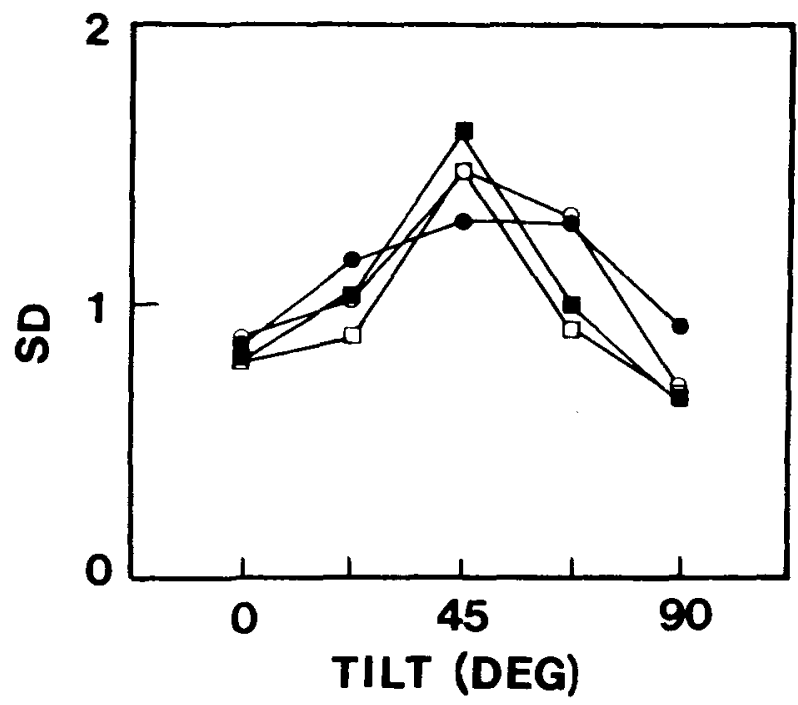

Figure 5. Estimated population standard deviations of the means in Figure 4 as a function of test edge orientation. Symbols are as for Figure 4.

\section{Results}

The results of Experiments 3 and 4 are plotted together in Figure 4, which shows mean illusions obtained for single angles (circles) and Bourdon displays (squares) for both solid and outline figures (filled and open symbols, respectively). Separate standard error bars are shown for each experiment. Figure 5 presents the standard deviations of the means in Figure 4, and demonstrates the wellestablished fact that precision is greatest at $0^{\circ}$ and $90^{\circ}$ with least precision at the oblique.

All 10 outline display means were significantly greater than zero. With $t(18)$ for outline angles and proceeding in order from $0^{\circ}$ to $90^{\circ}, t \mathrm{~s}=6.05,5.67,7.76,7.38$, and $5.48, p<.0005$ in every case. With $t(23)$ for outline Bourdon displays, $t \mathrm{~s}=6.69,7.11,6.57,6.81$, and 5.48 , and again $p<.0005$ in each case.

In contrast, for solid-angle displays, the mean illusions at $0^{\circ}$ and $90^{\circ}$ were not significant $(t \mathrm{~s}=-1.19$ and 0.38 , respectively, $p s>.05$ ). The other means, at $22.5^{\circ}, 45^{\circ}$, and $67.5^{\circ}$, were significant $(t=3.95, p<.005 ; t=$ 5.05, $p<.0005$; and $t=3.05, p<.01$ ). In the case of the solid Bourdon displays, only the mean at $67.5^{\circ}$ was significant $(t=-2.11, p<.05$, although on a one-tailed test, the $45^{\circ}$ mean also would have been significant).

As the above results might suggest, only the solidsingle-angle function in Figure 4 showed any significant trend and it was quadratic $[F(1,252)=17.13$, $p<.0005$ ]. As expected, the overall means induced by outline and solid displays differed both for single angles $[F(1,252)=204.97, p<.0005]$ and Bourdon figures $[F(1,322)=148.12, p<.0005]$.

\section{Discussion}

Experiments 3 and 4 demonstrated, as predicted, that outline effects, both single angle and Bourdon, are significant at $0^{\circ}$ and $90^{\circ}$. Although they may be slightly aug- 
mented at other orientations due to neural anisotropies (e.g., upper function in Figure 4), the large effects at $0^{\circ}$ and $90^{\circ}$ resulted in no significant quadratic trend.

Solid-angle and Bourdon illusions are not significant at $0^{\circ}$ and $90^{\circ}$, and tilting the figures away from these axes produces (rather than augments) the effects, presumably due to lower acuity at oblique orientations (Figure 5).

One puzzle remains: Although Wenderoth et al. (1986b) obtained single-angle illusions, both outline and solid, that were similar in magnitude to those reported here, with Bourdon figures they reported extremely small positive outline effects and large negative solid effects. In the present experiments, outline Bourdon illusions generally have been large and positive, whereas solid Bourdon illusions, especially in Experiment 4, were negative but very small. Part of the reason for variability in the magnitude of effects between experiments could be reflected in the fact that various proportions of subjects asked afterward whether all the edges were straight. However, such a "bias" seems unlikely to provide the sole explanation.

There is one essential difference between the present experiments and those reported by Wenderoth et al. (1986b). In the latter, the comparison chevrons were $1.2^{\circ}$ of visual angle from the judged edge, whereas we used a distance of $0.6^{\circ}$. The reason for this change was that observations by the first author had indicated that when the distance is larger, it is difficult to do the task by simultaneously matching the two edges. Rather, one tends to look at the test edge, then move the eyes to the comparison figure. The shorter distance enabled a more straightforward and simultaneous judgment of upper and lower parallelism, and it was already known that a distance of $0.4^{\circ}$ was sufficient to avoid interactions between inducing and comparison components (Carpenter \& Blakemore, 1973; O'Toole \& Wenderoth, 1977).

One possible effect of changing the distance of the comparison chevron is to change the nature of the judgment. When the comparison stimulus is close, the subject may make local matches of chevron to test edge that reflect the point-to-point apparent orientations of the test-edge segments. When the comparison stimulus is distant, the subject may make a more global judgment that involves judging the test edge in the context of the inducing edge and the overall mean orientations of the angles (their bisectors).

If this analysis is sound, it predicts that, when the test edge is straight, a systematic increase in the separation of comparison and test stimuli will increase solid Bourdon effects (i.e., make them more negative) but decrease outline Bourdon effects (i.e., make them less positive, or more negative, also). Experiment 5 tested this hypothesis.

\section{EXPERIMENT 5}

\section{Method}

Apparatus and Procedure. The apparatus and procedure were the same as those used in the previous experiments.
Stimuli. All Bourdon displays, outline and solid, had test edges oriented $67.5^{\circ}$ with $12.5^{\circ}$ acute angles. All dimensions were as in the previous experiments, except that the distance between the test edge and the comparison line was $0.3^{\circ}, 0.6^{\circ}, 0.9^{\circ}$, or $1.2^{\circ}$ of visual angle. Thus, there were 4 separations $\times$ outline, solid, pretest $\times 4$ starting positions $=48$ conditions, completed in random order by each subject.

Subjects. Fifteen subjects were drawn from the same population as those in the earlier experiments.

\section{Results}

The results, shown in Figure 6, were consistent with the prediction that increasing the separation of the matching chevron from the test edge would result in more negative solid illusions and less positive outline illusions. Contrast analysis showed that linear trend was significant for solid displays $[F(1,98)=5.43, p<.025]$. Quadratic and cubic trends were not significant $\left[F_{\mathrm{s}}(1,98)=0.68\right.$ and 0.006 , respectively, $p s>.05]$. For the outline displays, none of these trend components was significant, although linear came close $[F \mathrm{~s}(1,98)=3.68,1.67$, and 0.74 , respectively], but $t$ tests indicated that only the mean for the separation of $0.3^{\circ}$ was significantly different from zero, with $t(14)=3.19(p<.01)$. For the other means, $t=0.43,0.38$, and 0.33 (ps $>.05)$.

\section{Discussion}

Although the results of Experiment 5 generally were consistent with predictions, it perhaps is surprising that the outline effects at the $0.6^{\circ}, 0.9^{\circ}$, and $1.2^{\circ}$ positions were not significant. However, in all of these experiments, there has been a real problem of context effects: when edges are physically straight in all conditions, the inclusion of conditions that make this more or less obvious (e.g., outline figure with chevron at $1.2^{\circ}$ ) may affect results in other conditions. This could account for the non-

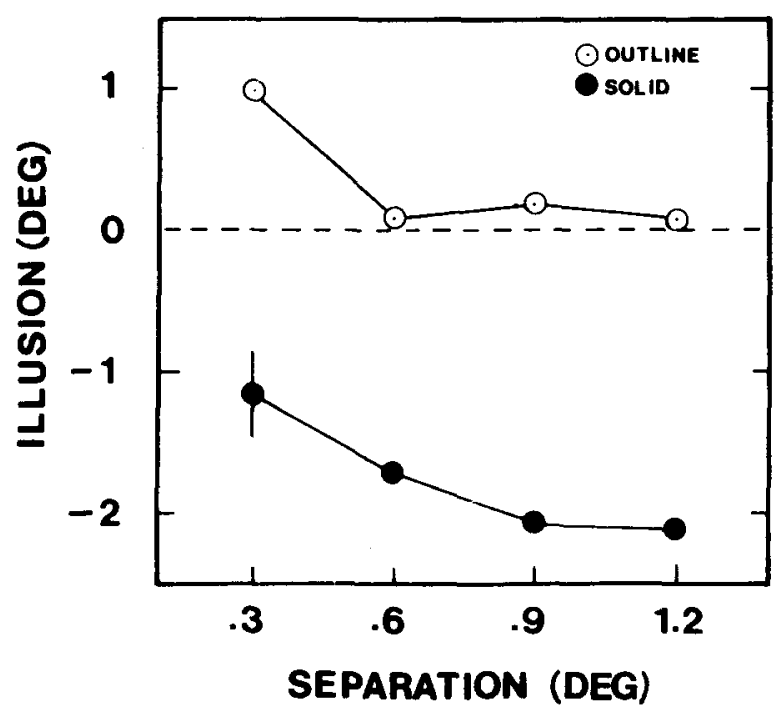

Figure 6. Mean outline and solid Bourdon illusions as a function of the orthogonal distance between the matching chevron and the test edge, Experiment 5. Error bar shows \pm 1 standard error. 
significant illusions. On the other hand, it may be, as suggested earlier, that in the $1.2^{\circ}$ condition subjects assess the whole figure rather than just its edge, and that this detracts from otherwise positive effects. There is a need to unconfound these two possible contributing factors, namely, response bias and global judgment.

\section{EXPERIMENT 6}

A final test of the hypothesis that solid-angle illusions occur due to a kind of acuity deficit, a failure to discriminate the test edge from the whole figure, might be to vary the sizes of the figures. It has been reported that simple tilt illusions do not vary systematically in magnitude as the arms of the acute angle are both increased in length (Wenderoth et al., 1986c). Acuity for parallelism, on the other hand, increases with line length (see Howard, 1982). In Experiment 6, subjects set chevrons $1.2^{\circ}$ away from test edges when both outline and solid Bourdon displays were derived from single angles with arm lengths of $1^{\circ}, 2^{\circ}$, and $3^{\circ}$ so that the total test edges were $2^{\circ}, 4^{\circ}$, or $6^{\circ}$ long, respectively. The predictions were that (1) negative solid illusions would be larger than positive outline illusions because the matching chevron was $1.2^{\circ}$ removed (Experiment 5), and (2) that increasing figure size would decrease solid-angle effects systematically but affect outline-angle effects in a nonsystematic fashion. All aspects of method were as for Experiment 5, and there were 20 subjects.

\section{Results and Discussion}

Figure 7 shows that the two predicted results occurred. First, when the figures were small, as in Experiment 5, outline effects were small and positive $(+0.46)$ whereas solid effects were large and negative $(-2.10)$, as predicted. Second, as outline-figure size increased, there was no significant linear or quadratic trend across the means $[F \mathrm{~s}(1,152)=0.37$ and 1.32 , respectively, $p s>.05]$. For solid figures, increasing figure size decreased illusions in linear fashion $[F(1,152)=20.58$, $p<.0005$, and quadratic trend was not significant $(F=$ $0.44)$.

\section{GENERAL DISCUSSION}

These six experiments strongly suggest that solid-angle illusions, including the Bourdon effect, are not neurally based effects in the way that outline tilt illusions are thought to be. The experiments suggest this conclusion because: (1) solid-angle illusions do not occur at all unless the test edge is straight, but this is not true of outline illusions (Experiments 1 and 2); (2) when test edges are vertical or horizontal, outline effects are reduced, presumably due to neural anisotropies, but solid-angle effects are eradicated completely (Experiments 1, 3, and 4); and (3) manipulations that decrease acuity for parallelism, such as increasing the separation of the matched edges or decreasing their length, have the effect

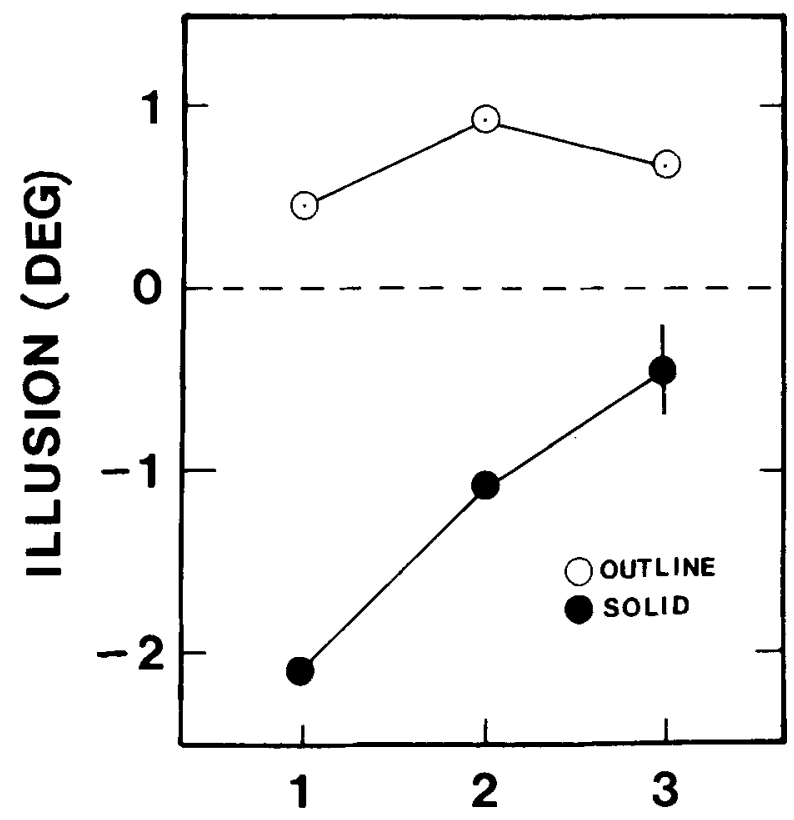

ARM LENGTH (DEG)

Figure 7. Mean outline and solid Bourdon illusions as a function of the length of each individual acute angle arm, Experiment 6. Error bar shows \pm 1 standard error.

of increasing solid-angle illusions but either decrease or have no effect on outline effects (Experiments 5 and 6).

These conclusions are not inconsistent with the hypothesis that orientation, position, and collinearity are neurally coded in parallel (Harris \& Calvert, 1985; Wenderoth et al., 1986b). Indeed, consistent with this hypothesis are the reduction in outline effects when edges are straight (Experiment 2) and the observation that a parallel chevron match to a solid Bourdon figure simultaneously appears parallel at all points yet appears insufficiently bent. So might be the finding that significant negative effects $\alpha c-$ cur with $90^{\circ}$ solid angles (Wenderoth et al., 1986b), a result not obtained with outline orientation effects.

Rozvany and Day (1980) obtained an asymmetry in Bourdon illusions as the figure tilted between $0^{\circ}$ and $90^{\circ}$ : the peak effect occurred at $67.5^{\circ}$ (not $45^{\circ}$ ), and the effects were slightly larger at vertical than at horizontal (although both were very small). A similar tendency appears in Figure 4, where the sole significant illusion occurred at $67.5^{\circ}$ and a slight negative illusion occurred at vertical.

This might be explained by the fact that when a Bourdon display is horizontal, its axis of symmetry is vertical so that it resembles a road disappearing to a flat horizon. Thus, the fact that symmetry about a vertical axis is more salient than symmetry about a horizontal axis might account for these asymmetric aspects of the data (Corballis \& Roldan, 1975; Goldmeier, 1972; Julesz, 1971; Mach, 1897, 1898; Rock \& Leaman, 1963; Schaller \& Harris, 1975). 
Finally, Rozvany and Day (1980) entertained the hypothesis that the Bourdon illusion might be in the same class of effects as negative Zöllner illusions, attraction effects that occur with very small angles and are thought to be due to summation of neural excitatory responses. The present experiments suggest that this is unlikely and that Bourdon effects are totally different from orientation illusions generally attributed to lateral inhibitory processes. Indeed, our analysis of the Bourdon effect leads us to predict that increasing the contrast of the display should decrease the effect, because increasing contrast would increase acuity for parallelism. Because of limitations of equipment, we were unable to conduct this experiment, but it would be instructive to do so, because increasing contrast of the inducing components of Zöllner and tilt illusions increases those effects (see Wenderoth et al., 1986c).

\section{REFERENCES}

APPELle, S. (1972). Perception and discrimination as a function of stimulus orientation. Psychological Bulletin, 78, 266-278.

BAuer, R., Dow, B. M., \& VAutiN, R. G. (1980). Laminar distribution of preferred orientations in foveal striate cortex of the monkey. Experimental Brain Research, 41, 54-59.

Bouma, H., \& ANDriessen, J. J. (1968). Perceived orientation of isolated line segments. Vision Research, 8, 493-507.

Campbell, F. W., KulikowskI, J. J. (1966). Orientational selectivity of the human visual system. Joumal of Physiology, 187, 437-445.

CARPENTER, R. H. S., \& BLAKEMORE, C. (1973). Interactions between orientations in human vision. Experimental Brain Research, 18, 287-303.

Corballis, M. C., \& Roldan, C. E. (1975). Detection of symmetry as a function of angular orientation. Joumal of Experimental Psychology: Human Perception \& Performance, 1, 221-230.

GoldmeIrr, E. (1972). Similarity in visually perceived forms. Psychological Issues, 8, Monograph 29, 1-136.

Harris, J. P., C CAlverT, J. E. (1985). The tilt after-effect: Changes with stimulus size and eccentricity. Spatial Vision, 1, 113-129.

Howard, I. P. (1982). Visual spatial orientation. New York: Wiley.

Judd, C. H., \& Courten, H. C. (1905). The Zöllner illusion. Psychological Review Monograph Supplement, 7, 112-139.
JuLesz, B. (1971). Foundations of cyclopean perception. Chicago: University of Chicago Press.

LeIBowITz, H., * ToFrey, S. (1966). The effect of rotation and tilt on the magnitude of the Poggendorff illusion. Vision Research, 6, 101-103.

МАС̆, E. (1897). Analysis of the sensations. Chicago: Open Court. MaCh, E. (1898). Popular scientific lectures. Chicago: Open Court.

MANSFIELd, R. J. W. (1974). Neural basis of orientation perception in primate vision. Science, 186, 1133-1134.

MANSFIELD, R. J. W., RoNNER, S. F. (1978). Orientation anisotropy in monkey visual cortex. Brain Research, 149, 229-234.

O'TOOLE, B. I., WENDEROTH, P. (1977). The tilt illusion: Repulsion and attraction effects in the oblique meridian. Vision Research, 17, 367-374.

OYamA, T. (1960). Japanese studies on the so-called geometrical-optical illusions. Psychologia, 3, 7-20.

OYama, T. (1975). Determinants of the Zöllner illusion. Psychological Research, 37, 261-280.

Rock, I., \& Leaman, R. (1963). An experimental analysis of visual symmetry. Acta Psychologica, 21, 171-183.

RozVANY, G. E., DAY, R. H. (1980). Determinants of the Bourdon effect. Perception \& Psychophysics, 28, 39-44.

SCHALLER, M. J., \& HarRis, L. J. (1975). 'Upright' orientation of forms change with subject age and with feature of form. Perception \& Psychophysics, 17, 179-188.

WALLACE, G. K. (1964). Measurements of the Zöllner illusion. Acta Psychologica, 22, 407-412.

Wenderoth, P., Johnson, M. (1984). The effects of angle-arm length on judgments of angle magnitude and orientation contrast. Perception \& Psychophysics, 36, 538-544.

Wenderoth, P., O’Connor, T., Johnson, M. (1986a). Evidence for a significant contribution of interactions between oriented line segments in the Tolansky version of the Poggendorff illusion. Perception \& Psychophysics, 39, 334-338.

Wenderoth, P., O'Connor, T., \& Johnson, M. (1986b). Expansion and contraction of outline and solid acute angles: Effects of angle magnitude, type of display, and the nature of the matching task. Perception \& Psychophysics, 39, 261-266.

Wenderoth, P., O'Connor, T., Johnson, M. (1986c). The tilt illusion as a function of the relative and absolute lengths of test and inducing lines. Perception \& Psychophysics, 39, 339-345.

WHITE, K. G. (1975). Orientation effects on contour interaction in the Zöllner illusion. Perception \& Psychophysics, 17, 387-392.

(Manuscript received May 28, 1986; accepted for publication August 4, 1986.) 